\title{
Mind as Energy
}

Dr Holly Pollard-Wright, independent researcher and CEO of Wild Ride Wildlife Services, has developed a mind which draws on principles from classic and quantum of transforming the mind behavioural and cognitive science. The mind is viewed energies of relationships, with no beginning and no end, that give rise to consciousness in an observer processing change or This theory has implications mind and the ethical treatment of all living beings. physics, ancient philosophies information from the universe.

hat is mind? Where does
it begin and where does it
end? What are the energies that constrain and transform minds? Can directed by effortful control? Do universa principles of physics apply to the minds of living beings? These are some of the questions that Dr Holly Pollard-Wright, CEO ofWild Ride Will Po Services Inc. and founder of Dark and Normal Matters MLC, sets out to answer in her Theory of principles from different scientific theor including concepts from Einstein's Theor, of Special Relativity, Dr Pollard-Wright proposes that scientific understright be applied symbolically, to understand the minds of living beings.

Essential concepts within this understanding of mind include the transformation of energies through interaction and relationship. Dr PollardWright puts forward three states of min a pure awareness state, a pure mental state, and a mental images state. Mind is proposed to be an "energy pure menta" it encounters through sensory signals.

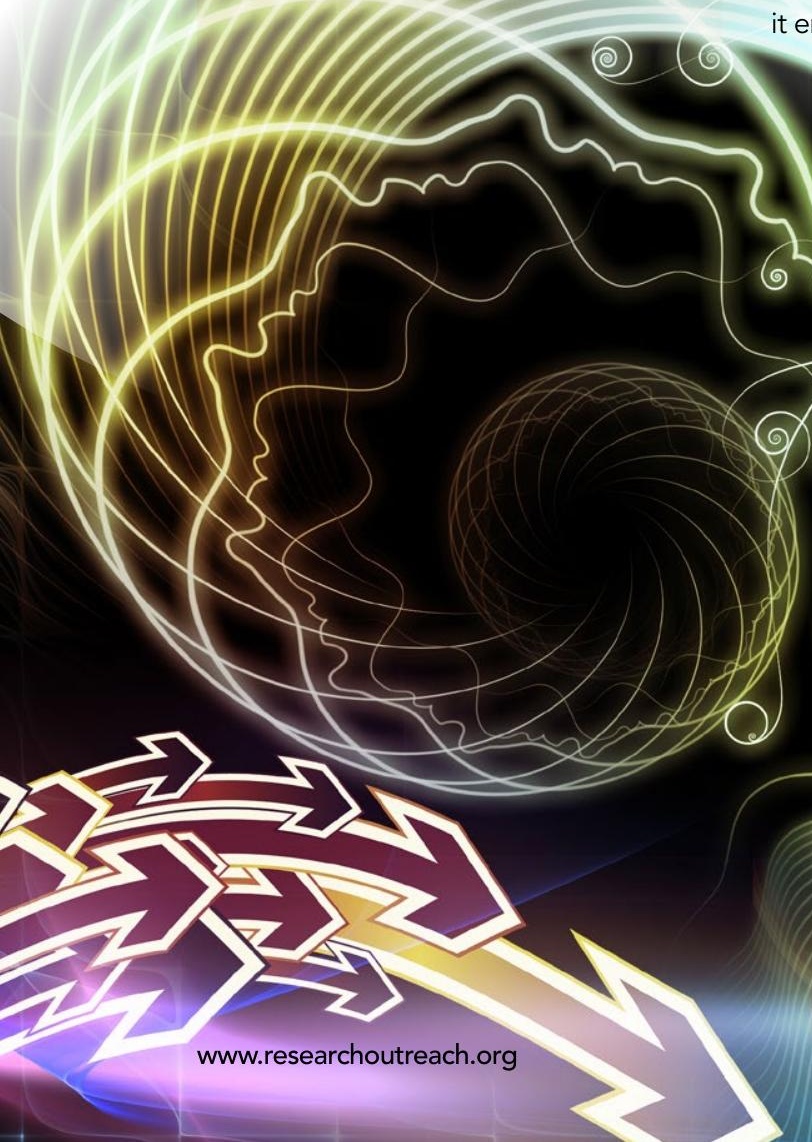

This is an unconscious activity of mind rein these signals are transmitted an observing ego which encodes the mordial feelings of stimuli s pleasant, unpleasant, and neutral. These encoded' records' are stored and 'whe which, in turn, become a stimulus for response when encountering stimu this 'Feeling of Knowing'. 'Feeling this 'Feeling of Knowing'. 'Feelings teroception - the sense from within the body - that regulates and drives behaviour. Inherent to this process are cyclical relationships involving interactions between the body (unconscious energy pure awareness of events), mind (energy mental images which create signals of the events), mind (energy pure mental which processes the signals' content), and mind (an observing ego that processes the

\section{MIND AS OBSERVING EGO} Dr Pollard-Wright proposes that mind embodies a pure mental state and a mental images state which is perceived by an observing ego. Mind thus incorporates three types of intelligence. Firstly, that of conscious events which and recorded as energy mental images. Second, unconscious intelligence which comprises events created by mind while detecting and processing information. Third, conscious intelligence created by actions and reactions of mind (pure mental energy and mental images energy) in recursive relationships with the energy pure awareness. Dr PollardWright says that this theory of mind is thus a "theory of everything that is the content of consciousness". Implicit in this theory is an understanding of the relationships of mind as bidirectional, reflexive and autopoietic. Mind thus becomes an observer or itself-or processing, regotini detecting, processing, recording, integrating and

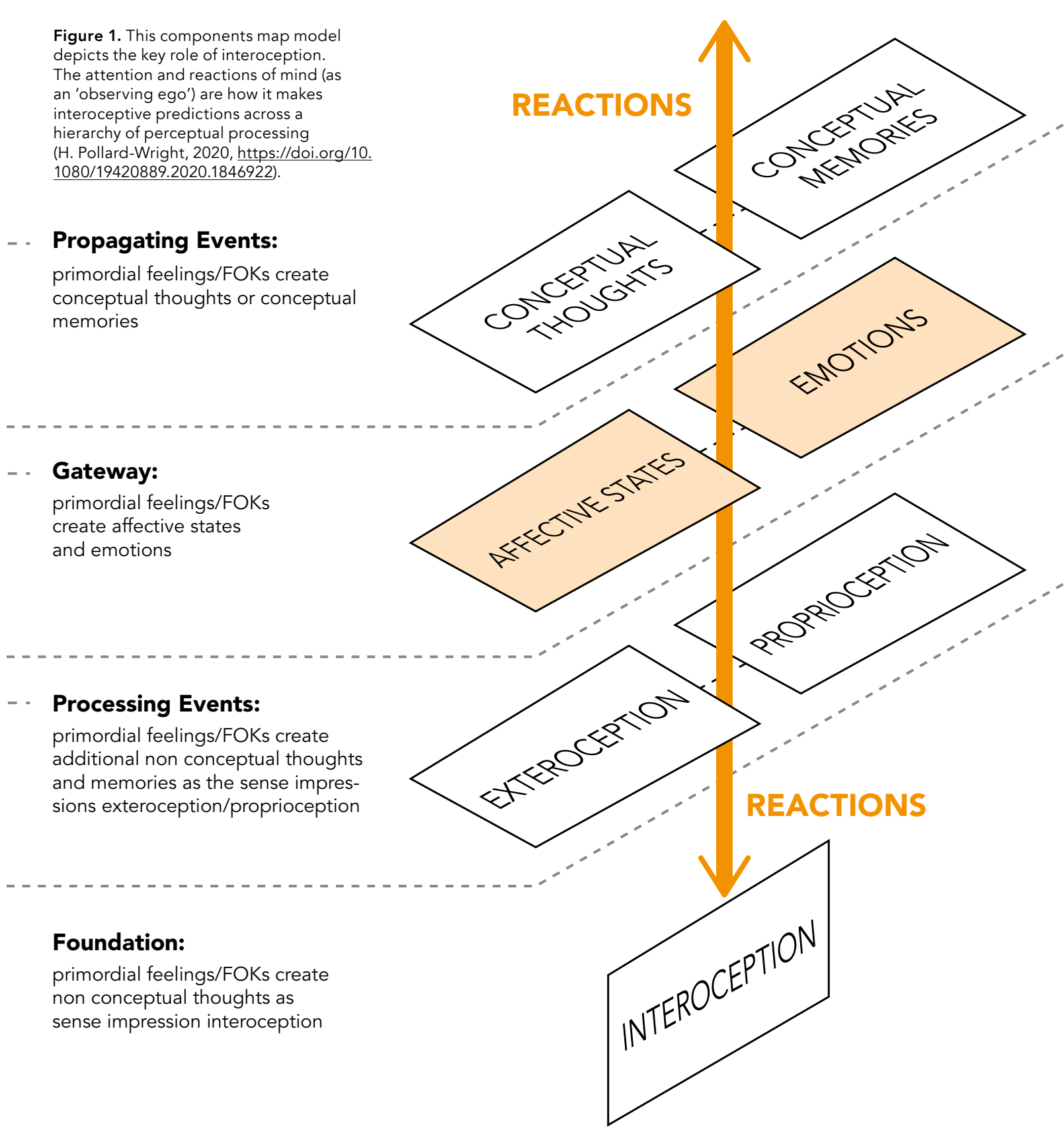

RELATIONSHIPS

OF MIND

Dr Pollard-Wright puts forward three

Dr Pollard-Wright states of mind: a pure awareness state, a pure mental state, and a mental

consciousness. This gives way to reactions that are autonomic
(reflexive) and model which sets out the different images state.

way to thoughts and memories mental content (see figure 1). The lower levels in this model also include exteroception (sensing stimuli outside the body) and proprioception (intuitive sense of the body's postion in physical space). Emotions and affective states map model, as states which act as a
These lower levels represent 'bottom-up' expectations or thoughts in this model of mind. Dr Pollard-Wright proposes that some of this content may represent 'the world of appearances'. Affective states automatically give rise to emotion and memories b, motions, thoughts those that are more considered and entall forethought. A causeming encoded records and this elationship is in essence cyclical and reflexive in relation to the energies and components of mind

INTEROCEPTION

Dr Pollard-Wright proposes that the 
forethought is linked to the level of wistivity to the bodily sensations. Thos

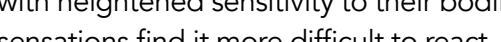
with forethought This can though be changed by altering the reaction from an mpulsive bottom-up driven response, to one which is more 'top-down' and involves using volition or 'will'.

\section{a

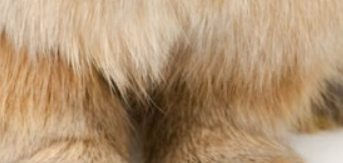 \\ as 4}

applying these principles of conscious intelligence to understanding fear in nimate lWing beings, Dr Pollard-Wrig proposes that the primordial feeling by brain electrochemical and synaptic neuronal activity which precedes ic making. This is the first 'moment of subjectivity or consciousness' This activity of mind produces a 'Feeling of Knowing' which when associated with interoceptive physical sensation within the body, may trigger an increased awareness of thought. This, in turn may cause mental suffering or pain for lving beings, such as the development of fear and phobias. Phobic behaviours are intense and excessive states of fear, often considered irrational by others observing the person displaying or expressing intense fear of a situation or object. These intense states of fear can result in an experience of panic, intense physiological reactions in living beings. These reactions can cause subjective distess with energy directed situation of fear. This suffering has its rigins in inter. Theptive 'Feelings of Knowing'. Dr Pollard-Wright proposes that these feelings have their origins in diverse regions of the peripheral nenous system, including the gut and the immu system of living beings, and associated electrochemical reactivity within the bra Unpleasant interoceptive experiences can, however, be important in alerting an individual to a potential threat or impending unpleasant experience. These interoceptive cues are learnt through experience and can be intense and overwhelming and difficult to 'over-ride', thus resulting in a feeling of panic and an impulse towards perceived harm avidance. Dr Pollard Wight says that described as a 'fight or flight response'.

\section{TRANSFORMING MIND}

Dr Pollard-Wright argues that it is possible

to transform an individual's interoceptive

intervention in transforming the mind's in to present with the ention Ulind process of obsenving the interoceptive pattern anchors the appraisal of the stimulus and provides the individual with a

MIND AS CONNECTED: EMPATHY Dr Pollard-Wright says that this theory of mind has application not only to understanding the minds of humans but also for understanding the consciousness of animals. She points out that we know that many animals show physical, behavioural and emotional responses, similar to that of humans when processing information and solving problems. Extending this theory of ming to animals offers a potential beginning and intelligen implications for the ethical treatment all living beings and the relationships between living beings. This theory of mind thus offers "a new perspective and respect for all living organisms on Earth".

This theory of mind has application not only to understanding the minds of humans but also for understanding the consciousness of animals.

experiences, through practices which incorporate mindfulness. Within this the first step towards understanding to be an obsener of 'bodily sensations' as patterns' (ie interoceptive patterns) which in turn evoke feelings. Identiffying the patterns which provoke emotional responses can enable an individual to feel more in control. Through this process of exploration by the individual of their interoceptive experiences which are causing neuropsychiatric or medical conditions, the individual can begin to disrupt the impulsive response to a stimulus. According to Dr PollardWright's model, an individual can learn to 'anchor' their responses by observing the bottom-up process of interoceptive patterns. The emphasis contrasts with a top down content focused approach cognitive rear, whe the focus is on cognitive restructuring. Within this moda

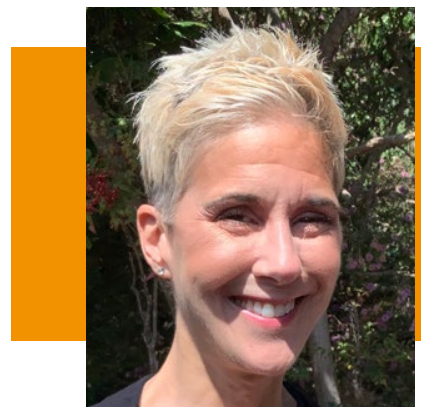

\section{Behind the Research}

Dr Holly Pollard-Wright

E: holly.pollard-wright@wildridevet.org E: holly@darkandnormalmatters.org

Research Objectives

Dr Pollard-Wright has formulated a transdisciplinary theory of mind.

\section{Detail}

Dr Holly Pollard-Wright, DVM, CCRP works clinically conprofit with a focus on providing surgical and medical care to injured and orphaned wildlife She is an independent researcher (IEEE NCIS) with peer-reviewed publications

with a wide range of interests.

\section{Collaborators}

Holly Pollard-Wright wishes to thank Lorelei McClure, Mark T. Wright, M.D., John E. Calamari, PhD., and Chris Skilbeck, PhD, for their comments that allowed the refining of this theory.

\section{Wild Ride \\ WILDLIFE SERVICES}

DARK \& NORMAL MATTERSW

\section{References}

Pollard-Wright, H. (2020). Electrochemical energy, primordial feelings, and feelings of knowing (FOK): Mindfulness-based intensention for interoceptive experience related to phobic https://doi.org/10.1016/j.mehy.2020.109909

Pollard-Wright, H. (2020). Interoception the Foundation for: Mind's Sensing of 'Self,' Physiological Responses, Cognit Integrative Biology. Available at: https://doi.org/10.1080/1942 0889.2020 .1846922

\section{Personal Response}

What are your hopes for changing the consciousness of

g to this theory, all living creatures with reflexively (i.e. automatically). Nonhuman individuals' emotional experiences arise from the cognitively contextualised perception of bodily states' changes and all living entities are intelligent, and those with brains and the central nervous system experience emotion and thus can suffer. As conceptualised by this theory, suffering signifies diversity in perception with a foundation of interoception that includes the reflexive experiencing of emotion. Accordingly, ibelieve that a radical shift commercial use and research, is in order.
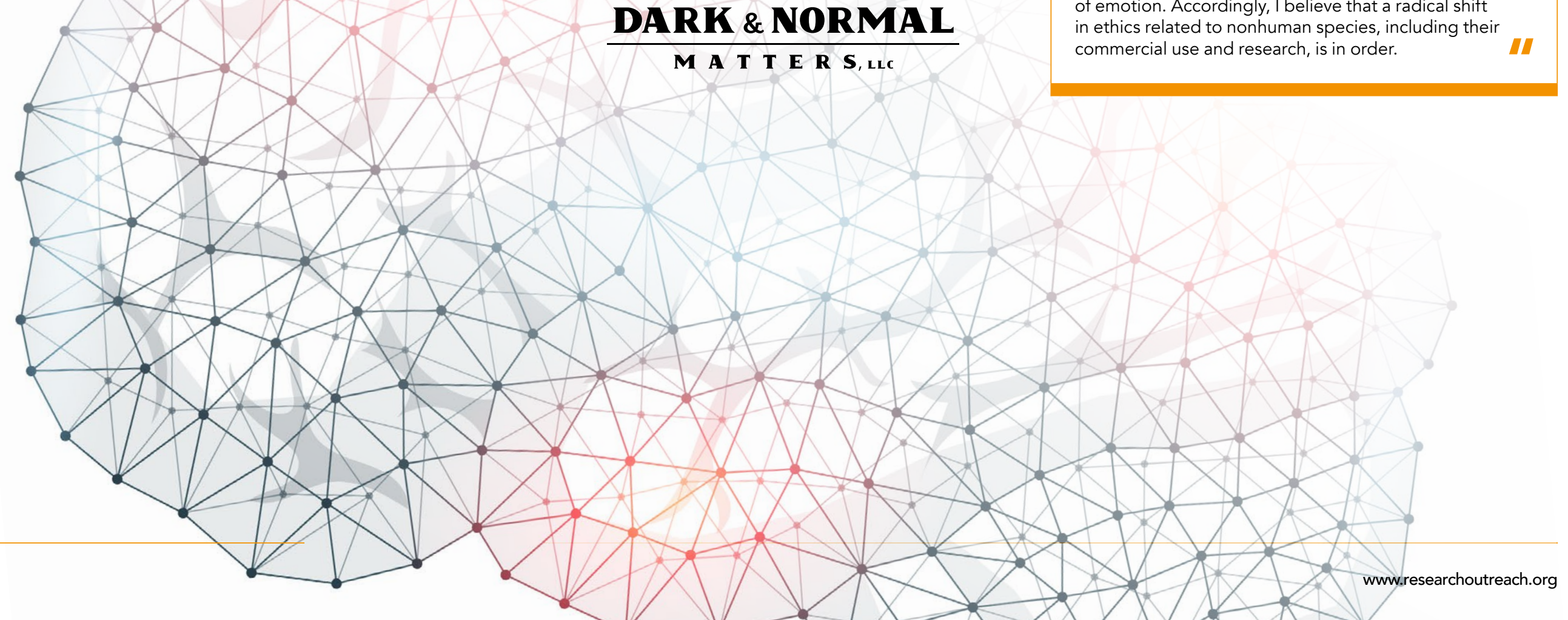